\title{
Parâmetros hemogasométricos e equilíbrio ácido-básico de cabritos nascidos de partos normais ${ }^{1}$
}

\author{
Diogo G. Camargo², Rodrigo Yanaka ${ }^{3}$, Fernanda Bovino ${ }^{2}$, Thales Bregadioli4 \\ Luiz C.N. Mendes ${ }^{5}$, Juliana R. Peiró ${ }^{5}$ e Francisco L.F. Feitosa ${ }^{5 *}$
}

\begin{abstract}
Camargo D.G., Yanaka R., Bovino F., Bregadioli T., Mendes L.C.N., Perió J.R. \& Feitosa F.L.F. 2012. [Blood gas parameters and acid-base balance of kids from normal delivery.] Parâmetros hemogasométricos e equilíbrio ácido-básico de cabritos nascidos de partos normais. Pesquisa Veterinária Brasileira 32(Supl.1):9-14. Departamento de Clínica e Cirurgia e Reprodução Animal, Faculdade de Medicina Veterinária de Araçatuba, Universidade Estadual Paulista, Rua Clóvis Pestana 793, Araçatuba, SP 16050, Brazil. E-mail: leydsonf@fmva.unesp.br

The extra-uterine life adaptation is a critical period for the newborn. Several changes occur in different organ systems including respiratory function and acid-base balance. The aim of this study was to hypothesize the occurrence of acidosis in newborn kids and obtain blood gas and acid-base parameters of kids in the first 24 hours of life. For this, 33 goat kids of both sexes were used. The blood samples were collected by jugular venipuncture immediately after birth, 5, 10,15 minutes and 24 hours of life. The blood pH, oxygen partial pressure $\left(\mathrm{pO}_{2}\right)$, carbon dioxide partial pressure $\left(\mathrm{pCO}_{2}\right)$, total carbon dioxide $\left(\mathrm{TCO}_{2}\right)$, bicarbonate concentration $\left(\mathrm{HCO}_{3}\right)$, base excess $(\mathrm{BE})$ and oxygen saturation $\left(\mathrm{SO}_{2}\right)$ were determined with a portable blood gas analyzer. Significant differences were observed between the moments in all variables of the kids blood gas values. The newborn kids had respiratory acidosis during the immediate post-partum period, normalizing at 24 hours of life.
\end{abstract}

INDEX TERMS: Blood gas, acid-base balance, acidosis, $\mathrm{pH}$, newborn, goats.

RESUMO.- A adaptação à vida extra-uterina é período crítico para o recém-nascido. Inúmeras alterações ocorrem em diferentes sistemas e órgãos, incluindo a função respiratória e o equilíbrio ácido-básico. 0 presente trabalho objetivou constatar a ocorrência de acidose em cabritos recém-nascidos e obter parâmetros gasométricos e ácido-básicos dos mesmos, até as 24 horas de vida. Para tanto, foram utilizados 33 cabritos, sem distinção de sexo. Amostras san-

\footnotetext{
${ }^{1}$ Recebido em 29 de junho de 2012.

Aceito para publicação em 18 de setembro de 2012.

2 Pós-Graduando do curso de Ciência Animal, área de Fisiopatologia Médica e Cirúrgica, Faculdade de Medicina Veterinária, Universidade Estadual Paulista (Unesp), Campus de Araçatuba, Rua Clóvis Pestana 793, Araçatuba, SP 16050-680, Brasil.

${ }^{3}$ Unidade Acadêmica de Garanhuns, Universidade Federal Rural de Pernambuco (UFRP), Avenida Bom Pastor s/n, Boa Vista, Garanhuns, PE 55292-270, Brasil.

${ }^{4}$ Bolsista de Iniciação à Pesquisa (FAPESP, Proc. 08/54029-1), Faculdade de Medicina Veterinária de Araçatuba, Unesp, Araçatuba, SP.

${ }^{5}$ Departamento de Clínica e Cirurgia e Reprodução Animal, Faculdade de Medicina Veterinária, Unesp, Araçatuba, SP. *Autor para correspondência: leydsonf@fmva.unesp.br
}

guíneas foram colhidas por venopunção jugular, imediatamente após o nascimento, aos cinco, dez e 15 minutos, e às 24 horas de vida, para determinação do $\mathrm{pH}$, pressão parcial de oxigênio $\left(\mathrm{pO}_{2}\right)$, pressão parcial de dióxido de carbono $\left(\mathrm{pCO}_{2}\right)$, dióxido de carbono total $\left(\mathrm{TCO}_{2}\right)$, concentração de bicarbonato $\left(\mathrm{HCO}_{3}\right)$, excesso de bases (BE) e a saturação de oxigênio $\left(\mathrm{SO}_{2}\right)$ utilizando-se analisador portátil de gases sanguíneos. Observaram-se diferenças significativas entre os momentos nos valores gasométricos dos cabritos em todas as variáveis estudadas. Os cabritos recém-nascidos apresentaram acidose respiratória no período pós-parto imediato, normalizando-se às 24 horas de vida.

TERMOS DE INDEXAÇÃO: Hemogasometria, equilíbrio ácido-básico, acidose, $\mathrm{pH}$, neonatos, caprinos.

\section{INTRODUÇÃO}

As prevalências de mortalidade perinatal de caprinos são bastante variáveis. Estudos indicam perdas de 10-60\% em sistemas extensivos de criação, e de 8-17\% em criações intensivas. Os prejuízos decorrentes da mortalidade perinatal incluem a perda dos recursos investidos nas matrizes, 
para iniciar e manter a gestação, além da redução do número de animais disponíveis para a venda e seleção (Ribeiro 1997, Maia \& Costa 1998).

Após o nascimento a sobrevivência do recém-nascido necessita de intensas mudanças nos padrões respiratório e circulatório. Esse processo adaptativo é dependente da ativação do eixo hipotálamo-hipófise-adrenal, sendo mediado, principalmente, pelo cortisol, um potente estimulador do metabolismo (Wood 1999). Porém, o mesmo pode ser suprimido nos casos de parto distócico, devido à possibilidade de ocorrência da síndrome asfixia/hipóxia neonatal (Ray et al. 1972).

0 exame hemogasométrico é de grande importância na avaliação do equilíbrio ácido-básico, pois pode fornecer informações fundamentais para o diagnóstico e o prognóstico de várias enfermidades dos bovinos (Sucupira \& Ortolani 2003). Dentre as disfunções do equilíbrio ácido-básico, a acidose metabólica é a mais comumente encontrada, caracterizada por diminuição do $\mathrm{pH}$ e dos valores de bicarbonato sanguíneo (Maruta \& Ortolani 2002, Kaneko et al. 2008).

0 sangue venoso é rotineiramente utilizado para a realização de hemogasometria por ser de mais fácil colheita do que o arterial, e por oferecer resultados confiáveis nos casos de acidose metabólica (Dibartola 1992). Sucupira \& Ortolani (2003) encontraram alta correlação nos valores de $\mathrm{pH}$, bicarbonado e déficit de base (BE) entre sangue venoso e arterial de bovinos, indicando que o sangue venoso pode refletir precisamente o equilíbrio ácido-básico.

Durante a gestação e o nascimento, o animal está sujeito a baixo suprimento de oxigênio. Os neonatos saudáveis sofrem de acidose discreta, sendo que os animais nascidos de partos laboriosos apresentam, invariavelmente, níveis significativamente mais baixos de pH sanguíneo (Wilson et al. 1976, Gardiner 1980).

As contrações uterinas e a ruptura das membranas fetais durante o parto normal causam distúrbios na circulação sanguínea útero-placentária, promovendo leve, porém transitória, acidose mista, considerada como fisiológica, com valor do pH sanguíneo por volta de 7,2 (Ravary-Plumioën 2009). Observa-se após a ruptura do cordão umbilical, durante a transição do fornecimento placentário de oxigênio para o estabelecimento da função respiratória, acidose metabólica e respiratória transitória discreta, por causa da glicólise anaeróbia em tecidos pouco perfundidos (Vaala \& House 2006). Esta condição é agravada pela reduzida capacidade respiratória do neonato, caracterizada por hipoventilação, que não garante a remoção do dióxido de carbono $\left(\mathrm{CO}_{2}\right)$ na mesma intensidade em que é produzido, aumentando a produção de ácido carbônico e, consequentemente, diminuição do pH sanguíneo (Piccione et al. 2006).

Para combater os desequilíbrios ácido-básicos o organismo utiliza três mecanismos principais, a saber : tamponamento químico, principalmente pelo bicarbonato, ajuste respiratório e excreção de íons pelos rins. Os sistemas tampão e respiratório atuam dentro de poucos minutos, ao contrário dos rins, que respondem lentamente ao excesso de ácido ou de base (Guyton \& Hall 2002, Houpt 2006).

Em bezerros é descrito que os desequilíbrios ácidos-basicos fisiológicos presentes logo após o nascimento são solucionados em torno de duas horas, no caso da acidose metabólica, e por volta de 24 a 48 horas para a acidose respiratória (Varga et al. 1998, Uystepruyst 2006). Tal condição pode ser devida à reversão fisiológica da acidemia sofrida durante o parto, em virtude do início da atividade respiratória e do mecanismo de filtração renal, obedecendo ao novo padrão circulatório e respiratório estabelecido pelo organismo (Haskins 1977, Benesi 1993).

Em bezerros recém-nascidos observou-se a influência etária nos valores hemogasométricos e do balanço ácido-básico nas primeiras 24 horas, tanto no sangue venoso quanto no arterial (Varga et al. 1999, Lisbôa et al. 2002, Gasparelli, 2007).

Yanaka (2012) constatou em caprinos recém-nascidos valores mais baixos de $\mathrm{pH}$ sanguíneo, caracterizando, assim, acidemia transitória, já que às 48 horas de vida a mesma já se apresentava normalizada. Piccione et al. (2006), estudando o sangue venoso de ovinos e caprinos durante a primeira semana de vida, observaram diferenças entre as espécies e entre os momentos em algumas das variáveis mensuradas.

0 presente trabalho teve como objetivo determinar os valores hemogasométricos e o equilíbrio ácido-básico de cabritos nascidos de partos normais.

\section{MATERIAL E MÉTODOS}

Foram utilizados 33 cabritos (14 machos e 19 fêmeas) da raça Bôer, oriundos de partos normais e sem qualquer tipo de auxílio. A determinação dos parâmetros vitais, hemogasométricos e avaliação do equilíbrio ácido-básico foi realizada nos seguintes momentos: zero hora (imediatamente após o parto), aos 5, 10 e 15 minutos, e às 24 horas de vida. Todos os cabritos permaneceram com as mães ingerindo colostro à vontade.

As colheitas de sangue foram feitas após anti-sepsia local, por punção da veia jugular, utilizando-se seringas apropriadas ${ }^{6}$ acopladas a agulha hipodérmica 25X0,7 mm. Quando presentes, o ar residual e as bolhas eram desprezados. A seringa era mantida selada e armazenada em recipiente térmico contendo gelo reciclável até o processamento da amostra.

Durante os momentos de colheita de sangue os animais foram avaliados por meio de exame físico, aferindo-se a freqüência cardíaca (FC), a freqüência respiratória (FR), a temperatura retal (TR) e, por fim, a coloração das mucosas visíveis, segundo as recomendações de Feitosa (2008).

A cada nascimento, as amostras foram colhidas e acondicionadas refrigeradas segundo Leal et al. (2010) até os 15 minutos de vida. A partir desse momento, foram imediatamente processadas seguindo-se a ordem das colheitas, ou seja, primeiro a amostra colhida à zero hora, seguida pelas de cinco, 10 e, por fim, a de 15 minutos de vida. No entanto, a amostra colhida às 24 horas de vida era processada imediatamente após sua obtenção.

Efetuou-se a determinação dos valores do $\mathrm{pH}$, pressão parcial de oxigênio $\left(\mathrm{pO}_{2}\right)$, pressão parcial de dióxido de carbono $\left(\mathrm{pCO}_{2}\right)$, dióxido de carbono total ( $\mathrm{tCO}_{2}$ ), concentração de bicarbonato $\left(\mathrm{HCO}_{3}{ }^{-}\right)$, excesso/déficit de bases (BE) e saturação de oxigênio $\left(\mathrm{sO}_{2}\right)$ em analisador clínico eletrônico portátili ${ }^{7}$, utilizando-se cartuchos ${ }^{8}$ específicos de acordo com as recomendações do fabrican-

\footnotetext{
${ }^{6}$ Drihep $^{\mathrm{TM}}$ A-Line ${ }^{\mathrm{TM}}$, Becton Dickinson Company, BD Brasil, São Paulo, Brasil.

7 i-Stat ${ }^{\circledR}$ Portable Clinical Analyzer, Abbott Laboratories, Abbott Park, Illinois, EUA.

${ }^{8}$ EG7+ Cartridge, Abbott Laboratories, Abbott Park, Illinois, EUA.
} 
Quadro 1. Média $(\overline{\mathrm{X}})$ e desvio padrão $(S)$ dos parâmetros hemogasométricos de cabritos até as 24 horas de vida. Araçatuba/SP, 2009

\begin{tabular}{|c|c|c|c|c|c|c|}
\hline \multirow[t]{2}{*}{ Variável } & \multirow{2}{*}{$\begin{array}{l}\text { Valores de } \\
\text { referência }\end{array}$} & \multicolumn{5}{|c|}{ Momento $(\bar{X} \pm S)$} \\
\hline & & 0 & 05 minutos & 10 minutos & $15 \mathrm{~m}$ & as \\
\hline $\mathrm{pH}$ & & & & & & \\
\hline & - & & 27,8 & & & \\
\hline & $40,0^{[b]}$ & 65,6 & 61,1 & 60,7 & 57,7 & \\
\hline & $25,6-29,6^{[c]}$ & $27,59 \pm 2,82^{\mathrm{b}}$ & $26,89 \pm 2,75^{\mathrm{ab}}$ & 26,53 & $25,83 \pm$ & 25,94 \\
\hline l/L) & $24,0^{[b]}$ & $25,82 \pm 2,70^{\mathrm{a}}$ & $25,18 \pm 2,61^{\mathrm{ab}}$ & $24,97 \pm 2,06^{\mathrm{ab}}$ & $24,36 \pm 2,69^{b}$ & $25,85 \pm 3,43^{a b}$ \\
\hline L) & $-4,0-+4,0^{[\mathrm{d}]}$ & $-1,47 \pm 3,61^{\mathrm{ab}}$ & $-2,14 \pm 3,58^{a b}$ & $-2,49 \pm 2,86^{b}$ & $-3,09 \pm 3,60^{b}$ & $0,44 \pm 2,96^{a}$ \\
\hline $\mathrm{sO}_{2} \mathrm{C} \%$ & 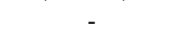 & $39,87 \pm 11,27^{\mathrm{a}}$ & $37,70 \pm 12,56^{\mathrm{ab}}$ & $31,50 \pm 12,76^{b}$ & $30,69 \pm 12,43^{b}$ & $48,79 \pm 13,49$ \\
\hline
\end{tabular}

Médias seguidas de letras diferentes na mesma linha diferem entre si ( $\mathrm{p}<0,05)$. (a) Meyer et al. 1995; (b) Thrall 2006; (c) Kaneko et al. 2008; (d) Haskins 1977.

te, sendo calibrado automaticamente antes do processamento das amostras. Adicionalmente, como controle de qualidade, utilizou-se o simulador eletrônico ${ }^{9}$ para verificar o funcionamento correto do equipamento antes do processamento. Os valores de $\mathrm{pH}, \mathrm{pO}_{2}$ e $\mathrm{pCO}_{2}$ foram ajustados pelo aparelho, de acordo com a temperatura retal de cada animal, aferida com termômetro clínico digital, como especificado pelo fabricante do aparelho.

Os dados foram avaliados quanto à normalidade pelo teste de Kolmogorov-Smirnov e, em seguida, submetidos à análise de variância com medidas repetidas no tempo (ANOVA). 0 contraste de médias foi feito pela diferença mímina significativa (d.m.s.) do teste de Tukey, ao nível de 5\% de probabilidade.

O presente experimento foi aprovado pela Comissão de Ética na Experimentação Animal de acordo com os princípios éticos na experimentação animal (COBEA), protocolo 2008-004818.

\section{RESULTADOS}

Os partos tiveram duração média de 36 minutos, entre o início das contrações e o nascimento do cabrito. Nos partos gemelares, a diferença entre o nascimento do primeiro para o segundo cabrito foi em média 15 minutos. Do total de partos observados, $68 \%(23 / 33)$ foram simples e $32 \%$ $(11 / 33)$ gemelares; cerca de $42,4 \%(14 / 33)$ dos animais nascidos eram machos e 57,6\% (20/33) fêmeas.

Os resultados da análise hemogasométrica dos cabritos, estão descritos no Quadro 1. Os valores de $\mathrm{pH}$ apresentaram elevação contínua desde o nascimento $(7,21 \pm 0,08)$ até as 24 horas de vida $(7,33 \pm 0,06)$, sendo que o valor encontrado ao final do primeiro dia de vida diferiu dos valores encontrados nos demais momentos.

Os valores observados para $\mathrm{pCO}_{2}$, diminuíram desde o nascimento $(65,61 \pm 8,61 \mathrm{mmHg})$ até as 24 horas de vida $(51,34 \pm 7,44 \mathrm{mmHg})$, sendo que o valor observado ao nascimento difere do demais momentos, e o valor, às 24 horas de vida, foi significativamente menor dentre todos os valores observados.

O bicarbonato é usualmente determinado pela mensuração do $\mathrm{TCO}_{2}$, sendo que ele corresponde por, aproximadamente, $95 \%$ do total deste parâmetro (Carlson \& Bruss 2008). Os valores obtidos para os parâmetros $\mathrm{HCO}_{3}$ e $\mathrm{TCO}_{2}$ apresentaram o mesmo padrão observado para os valores de $\mathrm{pCO}_{2}$, observando-se diminuição desde o nascimento até os 15 minutos de vida, de $25,82 \pm 2,70$ para $24,36 \pm 2,69 \mathrm{mmol} / \mathrm{L}$

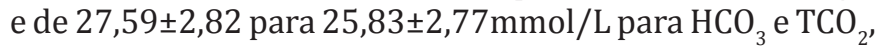

\footnotetext{
${ }^{9}$ i-Stat ${ }^{\circledR}$ Electronic Simulator, Abbott Laboratories, Abbott Park, Illinois, EUA.
}

Quadro 2. Média $(\overline{\mathrm{X}})$ e desvio padrão (S) de frequência cardíaca (FC), frequência respiratória (FR) e temperatura retal (TR) de cabritos até as 24 horas de vida. Araçatuba/SP, 2009

\begin{tabular}{lccccc}
\hline \multirow{2}{*}{ Variável } & \multicolumn{5}{c}{ Momento $(\overline{\mathrm{X}} \pm \mathrm{S})$} \\
\cline { 2 - 6 } & 0 hora & 5 minutos & 10 minutos & 15 minutos 24 horas \\
\hline FC $(\mathrm{bpm})$ & $159 \pm 29$ & $170 \pm 43$ & $175 \pm 36$ & $177 \pm 39$ & $171 \pm 34$ \\
FR (mpm) & $64 \pm 19$ & $65 \pm 15$ & $68 \pm 21$ & $65 \pm 15$ & $75 \pm 29$ \\
TR $\left({ }^{\circ} \mathrm{C}\right)$ & $38,7 \pm 0,8^{\text {bc }}$ & $38,0 \pm 1,1^{\text {ab }}$ & $37,6 \pm 1,3^{\text {a }}$ & $37,3 \pm 1,6^{\mathrm{a}}$ & $39,1 \pm 0,7^{\mathrm{c}}$
\end{tabular}

$\overline{\text { Médias seguidas }}$ de letras diferentes na mesma linha diferem entre si $(\mathrm{p}<0,05)$.

respectivamente, seguido por aumento às 24 horas de vida, $25,94 \pm 3,14 \mathrm{mmol} / \mathrm{L}$ e $25,85 \pm 3,43 \mathrm{mmol} / \mathrm{L}$ para $\mathrm{HCO}_{3}$ e $\mathrm{TCO}_{2}$, respectivamente, sugerindo, portanto, a estabilização do quadro metabólico.

Os valores obtidos para os parâmetros vitais estão descritos no Quadro 2. Houve diferença significativa entre os valores de TR ao nascimento quando comparados àqueles observados aos 10 e 15 minutos de vida, sendo que, os valores encontrados às 24 horas de vida, diferiram de todos os outros momentos avaliados.

0 exame das mucosas dos animais ao nascimento demonstrou que 27 neonatos apresentavam mucosas com coloração rósea-clara, cinco animais possuíam mucosas esbranquiçadas e somente um animal apresentou mucosa avermelhada. Às 24 horas de vida, a maioria dos animais já exibia mucosas normocoradas, com exceção dos animais que ainda apresentavam mucosas esbranquiçadas. Tal evolução demonstrou maior e melhor equilíbrio da hematose com o passar das horas e posterior ingestão do colostro.

\section{DISCUSSÃO}

Os esforços para reconhecer os valores de referência das variáveis hemogasométricas nos neonatos, assim como as variações que as mesmas podem apresentar no decorrer das quatro primeiras semanas de vida são amplamente justificados, pois fornecem, ao clínico, as informações necessárias destes parâmetros para adequada interpretação dos resultados.

Os valores médios obtidos para o $\mathrm{pH}$ sanguíneo de todos os animais recém-nascidos demonstraram a ocorrência de discreta acidose $(7,21)$, que, às 24 horas de vida, já se encontravam próximos aos valores de referencia, situados entre 7,35 e 7,45 (Meyer et al. 1995, Thrall, 2006). 
Se adotadas as considerações descritas por Thrall (2006), considerando os valores de referencia 7,4,40 mmHg e $24 \mathrm{mmol} / \mathrm{L}$ para $\mathrm{pH}, \mathrm{pCO}_{2}$ e $\mathrm{HCO}_{3}$, respectivamente, os baixos valores de $\mathrm{pH}$ observados ao nascimento, associados aos elevados valores de $\mathrm{pCO}_{2}$ e aos teores normais de $\mathrm{HCO}_{3}$ permitiu estabelecer o quadro de acidose respiratória e não de acidose mista, caracterizada pela diminuição do $\mathrm{pH}$, decréscimo nos teores de $\mathrm{HCO}_{3}{ }^{-}$e aumento da $\mathrm{pCO}_{2}$ (Houpt 2006, Thrall 2006).

No presente experimento, o rápido reconhecimento da acidose respiratória promoveu o início da resposta compensatória pela associação do incremento da ventilação e do tamponamento químico, reduzindo, consequentemente, a $\mathrm{pCO}_{2}$ em poucos minutos, e minimizando, dessa forma, a queda dos valores de $\mathrm{pH}$, com estabilização de seus valores, como verificado por Carlson \& Bruss (2008).

Apesar de os valores de $\mathrm{pH}$ continuarem abaixo dos padrões de referência, a tendência de estabilização do referido quadro foi evidenciada com o decorrer do tempo, ou seja, aos 15 minutos de vida, por serem maiores aos observados nos momentos pregressos; os de $\mathrm{pCO}_{2}$ diminuíram significativamente, e os valores de $\mathrm{HCO}_{3}$, mesmo apresentando diminuição significativa, ainda encontravam-se no intervalo de referência. Às 24 horas de vida os valores dos parâmetros indicavam que os neonatos já possuíam o quadro ácido-básico estabilizado.

No quadro de acidose respiratória é de se esperar, como resposta compensatória ao baixo $\mathrm{pH}$, o aumento dos níveis de bicarbonato acima dos valores considerados como normais, na tentativa de tamponamento químico do excesso de íons hidrogênio (Guyton \& Hall 2002). Contudo, como o componente metabólico está intimamente relacionado à capacidade renal de secretar íons hidrogênio e reter íons bicarbonato, o não incremento do íon bicarbonato no sangue dos cabritos utilizados ocorreu, possivelmente, devido à imaturidade ou menor capacidade de reabsorção/secreção renal durante as primeiras horas de vida e ao curto espaço de tempo que o organismo esteve frente ao desequilíbrio ácido básico. Tal resposta pode levar vários dias para atingir a sua capacidade máxima, mesmo em animais adultos (Houpt 2006, Carlson \& Bruss 2008). Portanto, a compensação metabólica só é observada em casos de acidose respiratória crônica (Carlson \& Bruss 2008).

Os valores de $\mathrm{pH}, \mathrm{pCO}_{2}$ e $\mathrm{HCO}_{3}$ encontrados no presente experimento, ao nascimento, estão de acordo com os relatados por Yanaka (2012) que também avaliou cabritos recém-nascidos provenientes de partos normais e observou, logo após o nascimento, valores de $7,21 \pm 0,06,66,50 \pm 8,0 \mathrm{mmHg}$ e $25,97 \pm 2,44 \mathrm{mmol} / \mathrm{L}$ para $\mathrm{pH}, \mathrm{pCO}_{2}$ e $\mathrm{HCO}_{3}$, respectivamente. Às 24 horas de vida, os valores estavam de acordo com os descritos por Piccione et al. (2006) 7,36 $\pm 0,06$ $(\mathrm{pH}), 44,6 \pm 5,1 \mathrm{mmHg}\left(\mathrm{pCO}_{2}\right)$ e $26,5 \pm 2,1 \mathrm{mmol} / \mathrm{L}\left(\mathrm{HCO}_{3}\right)$ para cabritos ao final do primeiro dia de idade, e bem próximos aos observados por Yanaka (2012) 7,30 $\pm 0,05(\mathrm{pH})$, $54,38 \pm 6,96 \mathrm{mmHg}\left(\mathrm{pCO}_{2}\right)$ e $25,52 \pm 2,85 \mathrm{mmol} / \mathrm{L}\left(\mathrm{HCO}_{3}\right)$ às 48 horas de vida, permitindo supor que as variações mais acentuadas no equilíbrio ácido-básico ocorrem nas primeiras 24 horas pós nascimento.
Isto posto, notou-se entre o nascimento e às 24 horas de vida, aumento médio do $\mathrm{pH}$, diminuição da $\mathrm{pCO}_{2}$ e diminuição com posterior aumento do valor de $\mathrm{HCO}_{3}$, como reflexos desta resposta compensatória. 0 mesmo padrão foi observado por Gasparelli (2007) em estudo realizado com bezerros, onde os mesmos apresentavam acidose respiratória ao nascimento e valores dentro dos limites de normalidade para a espécie ao final das primeiras 24 horas de vida, demonstrando, desta forma, a estabilização do equilíbrio ácido-básico durante o referido período.

Constatou-se que, logo após o nascimento, o déficit de base (BE) no sangue dos cabritos apresentou diminuição constante dos valores até os 15 minutos de vida; porém, esses valores mantiveram-se dentro dos limites de normalidade, de $-4 \mathrm{a}+4 \mathrm{mmol} / \mathrm{L}$ (Haskins 1977), em todos os momentos avaliados. Às 24 horas de vida houve aumento do valor encontrado, caracterizando, teoricamente, a estabilização do quadro metabólico e do equilíbrio ácido-básico.

Analisando-se os resultados referentes a $\mathrm{pO}_{2}$ e $\mathrm{sO}_{2}$ e, apesar destas variáveis terem sido obtidas no sangue venoso, observou-se pequena elevação dos valores de $\mathrm{pO}_{2}$, $31,5 \pm 8,03$ para $32,3 \pm 7,81 \mathrm{mmHg}$ e aumento mais acentuado dos valores de $\mathrm{s}_{2}, 39,87 \pm 11,27$ para $48,79 \pm 13,49 \%$, do nascimento até as 24 horas de vida. Este aumento ocorreu devido à melhora na capacidade respiratória dos animais, principalmente ao longo do primeiro dia de vida. Esta analogia encontra suporte nas observações feitas em bovinos (Aurich et al. 1993) e ovinos (Padbury et al. 1985, Padbury et al. 1987), que, durante o parto e principalmente após o rompimento do cordão umbilical, ocorre ativação do sistema simpático adrenal, com aumento na liberação de catecolaminas na primeira hora de vida, influenciando no aumento das frequências cardíaca e respiratória, na capacidade da função pulmonar, na produção de surfactante e na diminuição da produção de fluido pulmonar. A reabsorção do líquido pulmonar das vias aéreas durante as primeiras 24 horas pós-parto dos bezerros também contribui para a progressiva alteração da mecânica pulmonar (Varga et al. 2001).

De acordo com os resultados apresentados no Quadro 2, os valores médios encontrados no presente trabalho para a FR, até os primeiros 15 minutos de vida, encontravam-se dentro do intervalo de normalidade para animais desta espécie citado por Smith (2006), entre 40 e $65 \mathrm{mpm}$, bem acima dos valores citados como de normalidade, de 20$30 \mathrm{mpm}$, para animais adultos, por Feitosa (2008), e semelhante aos valores observados por Yanaka (2012), de 45$79 \mathrm{mpm}$, em cabritos neonatos ao nascimento.

Os valores médios encontrados para a FR, apesar de não apresentarem diferença estatística entre os momentos avaliados, apresentaram aumento, principalmente às 24 horas de vida. Tal aumento demonstra o papel desse parâmetro na estabilização do equilíbrio ácido-básico dentro do intervalo de normalidade com o decorrer do tempo, na tentativa de eliminar o excesso de $\mathrm{CO}_{2}$ produzido. $\mathrm{O}$ aumento da frequência respiratória aliado ao sistema tampão dos líquidos corporais aumenta a remoção de $\mathrm{CO}_{2}$, diminuindo, portanto, a formação de acido carbônico, acarretando, consequentemente, no aumento do pH sanguíneo (Verlander 2004). 
Os valores médios encontrados para a FC situavam-se acima dos intervalos de normalidade citados por Smith (2006), de 120-160 bpm, por Feitosa (2008), de 95-120 bpm e próximo ao citado por Yanaka (2012), de 119-191 bpm.

Foram constatados valores que estão compreendidos abaixo do intervalo normal de variação, de $38,8-40,2^{\circ} \mathrm{C}$, descrito por Feitosa (2008). Todavia, às 24 horas de vida, os valores encontrados estavam dentro da normalidade. Feitosa (2008) relatou que a idade influi sobre a temperatura retal. Os animais mais jovens têm maiores valores de temperatura, em virtude da menor capacidade reguladora, pois o centro termorregulador dos mesmos não está completamente desenvolvido, e do elevado metabolismo que esses animais apresentam.

Os resultados encontrados abaixo dos valores descritos na literatura podem ser explicados pela baixa temperatura ambiente que se fez presente durante as parições eutócicas, uma vez que as parições se deram no período de inverno e, grande parte delas, durante o período noturno. Animais jovens normalmente perdem mais calor para o ambiente devido à sua elevada superfície corpórea em relação à sua massa, uma vez que a troca de calor com o ambiente é dependente da condutância térmica, da área de superfície e do gradiente de temperatura (Robertshaw 2006).

Outro fator que possivelmente colaborou com a excessiva perda de calor foi a elevada frequência respiratória apresentada, na tentativa de eliminar o excesso de gás carbônico produzido, corrigindo, assim, o equilíbrio ácido básico. $\mathrm{O}$ ar atmosférico mais frio, ao entrar em contato com as mucosas das porções superiores do trato respiratório, leva ao resfriamento do sangue e, consequentemente, diminuição da temperatura do animal (Guyton \& Hall 2006, Robertshaw 2006).

\section{CONCLUSÃO}

Os cabritos neonatos provenientes de partos normais apresentam quadro de acidose respiratória transitória, com estabilização do equilíbrio ácido-básico no decorrer das primeiras 24 horas de vida.

Agradecimentos.- À Fundação de Amparo à Pesquisa do Estado de São Paulo (FAPESP), pelo apoio financeiro para a realização desse projeto (Proc. 08/53294-3).

\section{REFERÊNCIAS}

Aurich J.E., Dobrinski I., Petersen A., Grunert E., Rausch W.D. \& Chan W.W. 1993. Influence of labor and neonatal hypoxia on sympathoadrenal activation and methionine enkephalin release in calves. Am. J. Vet. Res., 54:1333-1338.

Baccari Júnior F. 1990. Métodos e técnicas de avaliação da adaptabilidade dos animais às condições tropicais. Anais Simpósio Internacional de Bioclimatologia Animal nos Trópicos, Brasília. Embrapa-DIE, Fortaleza/ CE, p.9-17.

Benesi F.J. 1993. Síndrome asfixia neonatal nos bezerros: importância e avaliação crítica. Arqs Esc. Med. Vet. UFBA 16(1):38-48.

Carlson G.P. \& Bruss M. 2008. Fluid, electrolyte and acid-base balance, p.529-559. In: Kaneko J.J. (Ed.), Clinical Biochemistry of Domestic Animals. $6^{\text {th }}$ ed. Academic Press, San Diego.

Dibartola S.P. 1992. Fluid Therapy in Small Animal Practice. W.B. Saunders, Philadelphia. 720p.
Feitosa F.L.F. 2008. Semiologia Veterinária: a arte do diagnóstico. $2^{a}$ ed. Roca, São Paulo. 807p.

Gardiner R. 1980. Cerebral blood flow and oxidative metabolism during hypoxia and asphyxia in the new-born calf and lamb. J. Physiol. 305:357376.

Gasparelli E.R.F. 2007. Determinação da atividade sérica de enzimas hepáticas e da concentração de uréia e creatinina, cortisol e imunoglobulina $G$ e dos valores hemogasométricos de bezerros da raça Nelore oriundos de fertilização in vivo (FV) e fertilização in vitro (FIV). Dissertação de Mestrado em Ciência Animal, Curso de Medicina Veterinária, Universidade Estadual Paulista, Araçatuba. 73p.

Guyton A.C. \& Hall J.E. 2002. Tratado de Fisiologia Médica. Guanabara Koogan, Rio de Janeiro.

Haskins S.C. 1997. An overview of acid-base physiology. J. Am. Vet. Med. Assoc. 170:423-428.

Houpt T.R. 2006. Equilíbrio ácido-básico, p.147-160. In: Reece W.O. (Ed.), Dukes, Fisiologia dos Animais Domésticos. 12ª ed. Guanabara Koogan, Rio de Janeiro.

Kaneko J.J., Harvey J.W. \& Bruss M.L. 2008. Clinical Biochemistry of Domestic Animals. Academic Press, New York, p.904.

Leal M.L.R., Soares P.C., Cyrillo F.C. \& Benesi F.J. 2010. Influence of refrigeration on blood gas parameters of caprine venous blood. Braz. J. Vet. Res. Anim. Sci. 47(2):105-110.

Lisbôa J.A.N., Benesi F.J., Leal M.L.R. \& Teixeira C.M.C. 2002. Efeito da idade sobre o equilíbrio ácido básico de bezerras sadias no primeiro mês de vida. Braz. J. Vet. Res. Anim. Sci. 39:136-142.

Maia M.S. \& Costa A.N. 1998. Influência da amamentação sobre a sobrevivência de cabritos ao desmame. Anais da $35^{a}$ Reunião Anual da Sociedade Brasileira de Zootecnia, Botucatu, SP, p.217-219.

Maruta C.A. \& Ortolani E.L. 2002. Susceptibilidade de bovinos das raças Jersey e Gir à acidose láctica ruminal. II. Acidose metabólica e metabolização de lactato-L. Ciência Rural 32:61-65.

Meyer D.J., Coles E.H. \& Rich L.J. 1995. Medicina de Laboratório Veterinária: interpretação e diagnóstico. Roca, São Paulo. 308p.

Padbury J., Agata Y., Ludlow J., Ikegami M., Baylen B. \& Humme J. 1987. Effect of fetal adrenalectomy on catecholamine release and physiologic adaptation at birth in sheep. J. Clin. Invest., 80:1096-1103.

Padbury J.F., Polk D.H., Newnham J.P. \& Lam R.W. 1985. Neonatal adaptation: greater sympathoadrenal response in preterm than full-term fetal sheep at birth. Am. J. Physiol. 248:443-449.

Piccione G., Costa A., Bertolucci C., Borruso M., Pennisi P. \& Caola G. 2006. Acid-base balance modifications in the lamb and goat kids during the first week of life. Small Rumin. Res. 63:304-308.

Ravary-Plumioën B. 2009. Resuscitation procedures and life support of the newborn calf. Revue Méd. Vét. 160(8/9):410-419.

Ray D.E., Hansen W.J. \& Theurer C.B. \& Scott G.H. 1972. Physical stress and corticoid levels of stress. J. Anim. Sci. 34:900.

Ribeiro S.D.A. 1997. Caprinocultores: criação racional de caprinos. Nobel, São Paulo. 205p.

Robertshaw D. 2006 Regulação da temperatura e o ambiente térmico, p.897-908. In: Reece W.O. (Ed.), Dukes, Fisiologia dos Animais Domésticos. $12^{a}$ ed. Guanabara Koogan, Rio de Janeiro.

Smith B.P. 2006. Medicina Interna de Grandes Animais. 3aㅗ d. Manole, Barueri. 1728p.

Sucupira M.C.A. \& Ortolani E.L. 2003. Uso de sangue arterial e venoso no exame do equilíbrio ácido-básico de novilhos normais ou com acidose metabólica. Ciência Rural 33(5):863-868.

Thrall M.A. 2006. Hematologia e Bioquímica Clínica Veterinária. Roca, São Paulo. 582p.

Uysterpruyst C. 2006. Physiologie et réanimation néonatales duveau. Point Vét. 37:100-105.

Vaala W.E. \& House J.K. 2006. Adaptação, asfixia e reanimação perinatais, p.266-276. In: Smith B.P. (Ed.), Medicina Interna de Grandes Animais. 3a ed. Manole, Barueri. 1728p. 
Varga J., Mester L., Börzsönyi L. Leukeux P. \& Szenci O. 2001. Improved pulmonary adaptation in newborn calves with postnatal acidosis. Vet. J. 162:226-232.

Varga J., Mesrter L., Börzsönyi L., Erdesz C.S., Vari A., Körmöczi P.S. \& Szenci 0. 1999. Adaptation of respiration to extrauterine life healthy in newborn calves. Reprod. Dom. Anim. 34:377-379.

Varga J., Szenci O., Dufrasne I., Börzsönyi L. \& Lekeux P. 1998. Respiratory mechanical function in newborn calves immediately postpartum. Vet. J. 156:73-76.

Verlander J.W. 2004. Equilíbrio ácido-básico, p.471-480. In: Cunnhingam
J.G. (Ed.), Tratado de Fisiologia Veterinária. Guanabara Koogan, Rio de Janeiro. 579p.

Yanaka R., Camargo D.G., Bovino F., Santos W.A. \& Feitosa F.L.F. 2012. Evaluation of blood gas and acid-base balance of goat kids from birth to 75 days of life. Anais $26^{\text {th }}$ World Buiatrics Congress, Lisboa, Portugal, p.307. (Resumo) Wood C.E. 1999. Control of parturition in ruminants. J. Reprod. Fertil. 54:115-126.

Wilson G.D.A., Hunter R.J.T., Derrick G.H., Aitken W.M. \& Kronfeld D.S. 1976. Fetal and maternal plasma electrolytes, blood gases and $\mathrm{pH}$ in dairy cows during late gestation. J. Dairy Sci. 60:1110-1116. 